Mahuya Deb, Prabjot Kaur and Kandarpa Kumar Sarma*

\title{
Fuzzy Approach to Decision Support System Design for Inventory Control and Management
}

https://doi.org/10.1515/jisys-2017-0143

Received April 9, 2017; previously published online August 25, 2017.

\begin{abstract}
The ubiquitous nature of inventory and its reliance on a reliable decision support system (DSS) is crucial for ensuring continuous availability of goods. The DSS needs to be designed in a manner that enables it to highlight its present status. Further, the DSS should be able to provide indications about subtle and large-scale variations that are likely to occur in the supply chain within the context of the decision-making framework and inventory management. However, while dealing with the parameters of the system, it is observed that its operations and mechanisms are surrounded by uncertain, imprecise, and vague environments. Fuzzy-based approaches are best suited for such situations; however, these require assistance from learning systems like artificial neural network (ANN) to facilitate automated decision support. When ANN and fuzzy are combined, the fuzzy neural system and the neuro-fuzzy system (NFS) are formulated. The model of the DSS reported here is based on a framework commonly known as adaptive neuro-fuzzy inference system (ANFIS), which is a version of NFS. The configured model has the advantages of both the ANN and fuzzy systems, and has been tested for the design of a DSS for use as part of inventory control. In this work, we report the design of an ANFIS-based DSS configured to work as DSS for inventory management. The system accepts demand as input and generates procurement, ordering, and holding cost to control production and supply. The system deals with a certain profitability rating required to quantify the changes in the input and is combined with the day-to-day inventory records and demand-available cycle. The effectiveness of the system has been checked in terms of number and types of membership used, accuracy generated, and computational efficiency accounted by the computation cycles required.
\end{abstract}

Keywords: Inventory control, DSS framework, ANFIS, decision making.

\section{Introduction}

Inventory management and control are crucial elements in all segments of the economy. The traditional models of inventory design are built on crisp parameters where decision support is between two extremes. However, practical situations of inventory control demand that there be flexibility to handle uncertain situations. Most of these situations are closely entwined with the demand-supply chain on the one hand and control of the cost of production on the other. The various elements associated with how much to order are normally concerned with inventory costs and lot-sizing models. The inventory cost covers ordering, procurement, holding, and shortage. Inventory models are utilized to minimize operational costs and facilitate certain give-and-take approaches related to investment and customer satisfaction. A decision support system (DSS) may be used to achieve such objectives. The term DSS was first coined by P. G. W. Keen, a British academician who defined DSS as a computerized mechanism along with its analytical tools and manager's judgment for smooth handling of decision-dependent situations like inventory control, as mentioned in Ref. [1]. Aengchuan et al. defined a DSS to be "a computer based information system that combines models and data

\footnotetext{
*Corresponding author: Kandarpa Kumar Sarma, Department of Electronics and Communication Technology, Gauhati University, Gopinath Bordoloi Nagar, Guwahati, Assam 781014, India, e-mail: kandarpaks@gmail.com, kandarpaks@yahoo.co.in

Mahuya Deb: Department of Mathematics, Birla Institute of Technology, Mesra, Ranchi, Jharkhand, India

Prabjot Kaur: Birla Institute of Technology, Ranchi, Jharkhand, India
} 
in an attempt to solve semi-structured and some unstructured problems with extensive user involvement." In many situations, DSS related to inventory control is deployed in situations where there are innumerable uncertainties in terms of buyer and seller behavior, factors linked with production and transportation, facilities for holding and storage, etc. Normal DSS approaches may not be sufficient to cover the entire gamut of operations [1]. This is because most traditional approaches consider the extreme limits of operations or conditions that are covered by crisp decision logic. As a result, the states within the operations or process limits remain unaddressed. In such cases, fuzzy-based approaches are best suited because these can keep an eye on minute and large-scale variations. DSS consists of components like inputs and user knowledge, while expertise of the management system acts as a viable support for effective decision making with regard to inventory policy decisions, as reported in Ref. [17]. However, the parameters that define the inventory control models are not always crisp and are found to be uncertain, ambiguous, and vague. Against this backdrop, fuzzy-based approaches formulated by Zadeh [24] are best suited to tackle the imprecise and uncertain parameters associated with a DSS of an inventory control system. However, fuzzy systems cannot operate alone and require help from learning systems like artificial neural network (ANN) to provide automated decision support. Therefore, fuzzy-based DSS implemented using ANN is a reliable element of DSS as part of inventory control and management. Several literature reports have focused on such possibilities. A few are mentioned in Refs. [1, 6, 8-17, 22, 23].

Over the years, learning-based systems like ANN have been established as suitable options for a range of estimation and prediction-based systems, including DSS, because these can capture the intricate linkages between input and output data without bothering at all about the model involved, retain the know-how, and use it subsequently. In the adaptive neuro-fuzzy inference system (ANFIS) form, the advantages of the ANN and fuzzy systems are combined that can adaptively track the variations in the environment and dynamically update the network parameters. As a result, a reliable inference system is formulated that can learn finite to large-scale variations and provide effective decision support in an inventory management system. The decision support is learning aided, which is derived from the benefits of using ANN, which learns from process data and retains and applies them subsequently. This has been the basis of operation of learningaided systems that have established themselves as popular prediction tools for a range of applications.

Ahmed et al. [3] reported the use of ANN for image texture analysis. Use of ANN for protein structure prediction has been reported in Ref. [5]. Variations of ANN and fuzzy systems in different combinations have been applied in wireless channels and related data recovery applications, as reported in Refs. [4, 18, 19, 21]. For human-computer interactions, learning-based tools like ANN, fuzzy and derivatives, and related prediction tools have been applied and reported in Refs. [2, 7, 20]. It shows that learning-based tools like ANN, fuzzy, combinations of the former two, and derivatives have been applied to several real-world situations.

Various researchers, time and again, have incorporated fuzziness in inventory control models, like Chang [6], Park [14], Kacprzyk and Stanieski [10], Wee et al. [23], and Maiti and Maiti [13], to name a few. Even Paul and Azeem [16] used fuzzy logic for selecting the optimal number of shifts using inventory information, reliability of machines, and customer requirements. Various constraints like raw materials, demand, finished goods, etc., were used while trying to optimize the number of shifts. Lo [12] formulated a DSS for an integrated inventory model where the objective has been to minimize the joint total expected cost. Paul and Azeem [15] determined the optimal level of inventory using an ANN that is a function of product demand, material costs, setup, and holding cost. In Ref. [9], another finance problem has been effectively solved using an ANN model. In Ref. [11], Lin et al. report the formulation of a wafer fabrication process price by integrating an ANN and sequential quadratic programming. When ANN and fuzzy are combined, the fuzzy neural system and the neuro-fuzzy system are created. These two hybrid blocks acquire quantitative and qualitative abilities with learning, which enables them to form efficient DSS. Efendigil et al. [8] forecasted the incomplete demand information by artificial intelligence approaches using ANNs and ANFIS techniques. Also in Ref. [22], a successful implementation of ANFIS for long-term demand prediction of natural gas consumption has been reported. In Ref. [1], the fuzzy inference system (FIS) model in the form of an ANFIS has been reported. It has been used for an inventory system for both supply and demand. This model gave the best performance of total inventory cost saving by $>75 \%$ as compared to the stochastic economic order quantity (EOQ) model. 
However, none of the above works have focused on the design of a DSS based on ANFIS that is driven by intuitive and linguistic terms learning the variations in demand of certain goods and establishing their association with the related costs, as observed in the data set used for certain supply chain management.

The proposed DSS is expected to facilitate easy and smooth interaction with the human operators, with a decision-making process driven by intuitive and linguistic terms. A FIS is used with fuzzy forms of inputs and output variables converted to real-world format to make the system user-friendly. In this work, we report the design of a fast and reliable ANFIS-based DSS configured to work as part of an inventory management. The system accepts demand as input and generates procurement, ordering, and holding cost to control production and supply. The system deals with a certain profitability rating required to quantify the changes in the input and is combined with the day-to-day inventory records and demand-available cycle. The effectiveness of the system has been checked in terms of the number and types of membership (MF) used, accuracy generated, and computational efficiency accounted by the computation cycles required. The experiments are carried out for certain data acquired from a shopping complex distributed over 6 months. The results are also compared with that obtained from ANN formulated in two different forms. Compared to the work reported in Ref. [1], the proposed work shows a cost saving of $81 \%$ due to its speed of operation and accuracy of prediction. The organization of the paper is as follows: in Section 2, the formulated model of ANFIS-based DSS for inventory control finds a place. Section 3 discusses the experimental details and results. Section 4 exclusively mentions the conclusion of the paper.

\section{Proposed Model of ANFIS-Based DSS for Inventory Control}

The primary objective of the work is to formulate an ANFIS-aided DSS for inventory control and management, and demonstrate the cost savings achieved. The methodology involves collection of data of a certain supply chain that are integral to current economic systems, formulation of ANFIS-based DSS for inventory control, training and testing of the DSS, and analysis of the performance in terms of accuracy of prediction of demand and cost savings achieved compared to the traditional EOQ method. The proposed system may be described using a diagram shown in Figure 1. It is formed by an ANFIS that has a number of MFs, and its configuration is fixed after performing a range of experiments in terms of the number and types of MF used, accuracy generated, and computational efficiency accounted by the computation cycles required. The MFs are important in deriving the fuzzy inferences and capturing the finite variations in the input pattern. The speed of computation is required as it determines the response time required for providing a decision. The process logic involved in linking demand, which is the input, and the outputs, i.e. procurement, ordering, and holding costs, are outlined in Figure 1.

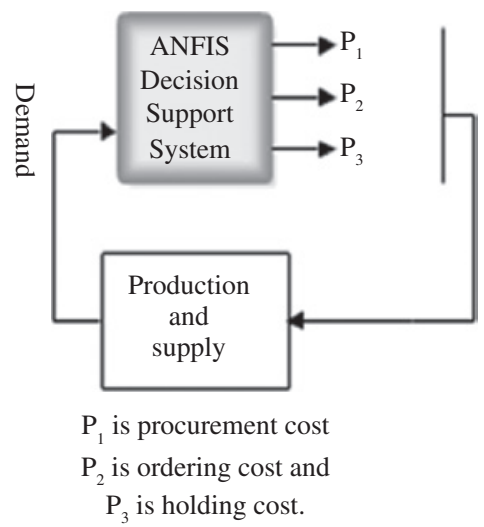

Figure 1: Process Logic of the Proposed Decision Support System for Inventory Control Using Fuzzy-Based Approach. 
The mechanism involves learning of the variations in demand of certain goods and establishing their association with the related costs observed in the data set used for the purpose. The work has two phases. First, the learning takes place. Next, the system is tested. During the learning phase, the input-output mapping is acquired by the ANFIS, which it tracks adaptively and identifies the discrimination boundaries despite the data having finer variations. The training-testing cycle is shown in Figure 2.

The learning is sustained till the objectives are achieved. To ascertain the state of learning, two variables are used: (i) the number of training cycles or epochs the ANFIS requires to attain the convergence goal and (ii) the accuracy generated in making the decision. These two aspects are linked with the numbers and types of MFs used with the ANFIS. This aspect is discussed in the next section. A major portion of the work is the design and configuration of the ANFIS. It is depicted in Figure 3.

ANFIS has been preferred as DSS in a range of applications. The fuzzy model involved can be configured either in terms of the Mamdani or Takagi, Sugeno, and Kang (TSK) representation. However, the TSK model is computationally more efficient than the Mamdani model and hence is popular in many applications. The selection of the model is based on the FIS.

The ANFIS architecture having five layers performs the primary task of decision making using a fuzzified back-propagation algorithm. The first layer deals with fuzzification. It adaptively converts the real-world inputs to fuzzy forms using a number of MFs. The common MF types include Bell and Gaussian forms. With greater number of MFs, the precision increases but it also demands more computational resources, including storage and processing cycles. A compromise is made between the number of MFs necessary and the

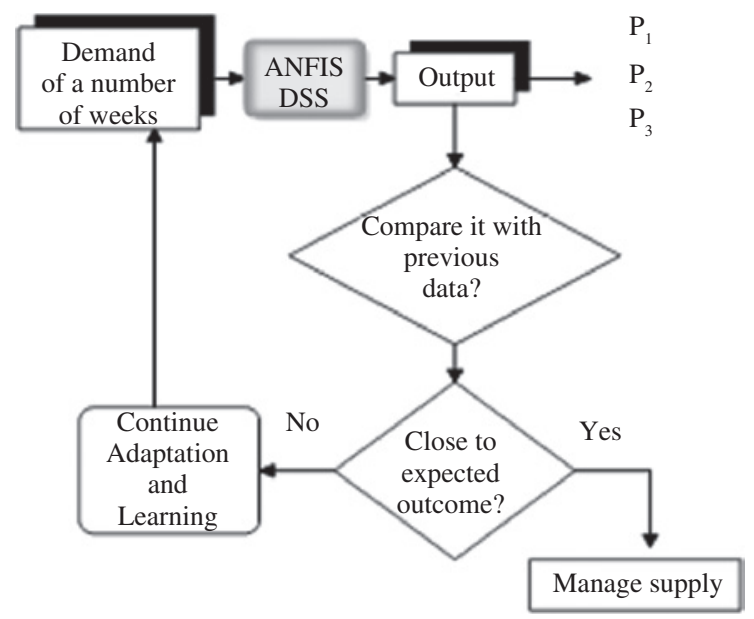

Figure 2: Training-Testing Configuration of the ANFIS-Based DSS.

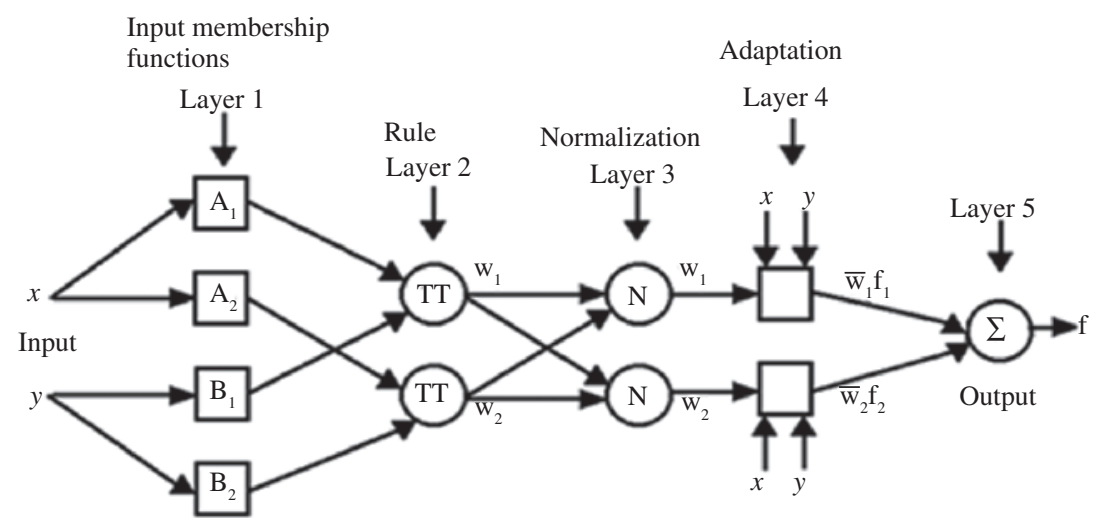

Figure 3: Different Layers of the ANFIS. 
accuracy acceptable. This is ascertained using a trial-and-error approach and a number of experiment cycles. The related details have been explained in the next section.

The second layer is responsible for generating the output of the first layer due to the fuzzy inputs. The nodes represent the firing or triggering ability of the network and formulate the rule set for the system. With more number of nodes, the accuracy increases; however, the system becomes time consuming. A balance between the number of nodes adopted in the second layer and the accuracy expected is derived from experimental results. Normalization takes place in the third layer. It expresses the value of a node in terms of the firing or triggering strength of the node scaled by the firing or triggering strength of all the nodes. For the fuzzy responses, adaptive update is done by the fourth layer. All of the learning takes place through this layer and involves the complete system, the data sets, and the class codes fixed for decision making. The last layer performs the task of summation of all the outputs. It generates the overall output of the network.

\section{Experimental Details and Results}

The results are derived using around 6 months of data of a certain supply chain. The data are categorized as outlined below with certain profitability rating required to quantify the changes in the input.

The primary block of the proposed system is the ANFIS. The ANFIS configured for the work with five layers has certain specifications, as summarized in Table 1.

Results are derived from the average of 10 trials for the epoch considered. Most reliable real-world experiences are provided by the Gaussian-curve membership functions, and as such they provide the most reliable real world to fuzzy conversion. It is therefore used for the fuzzification in the ANFIS layer. Ten such MFs give optimum results and hence form the basis of the ANFIS for training and testing. The cost function considered for training the ANFIS is the root mean squared error (RMSE). The system is trained using a data set for a commodity for several weeks. One such example is elaborated in Table 2.

Table 1: Parameter Specification.

\begin{tabular}{lll}
\hline Sl. No. & Description & Details \\
\hline 1 & Training, testing, and validation data & Data of a shopping complex for 6 months \\
2 & Variations in the data & $\pm 50 \%$ \\
3 & ANFIS & Five-layered structure \\
4 & ANFIS training & Fuzzy back-propagation \\
5 & Training qualitative measure & Least-square distance \\
6 & Average no. of epochs & $50-200$ \\
7 & No. of membership functions & 10 \\
8 & Types of membership function & Generalized Gaussian and Bell MFs \\
9 & Cost function for ANFIS & RMSE \\
10 & RMSE with 9 Gaussian MFs at 200 epochs & $1.7 \times 10^{-4}$ \\
\hline
\end{tabular}

Table 2: Example of Truncated Training Data for a Commodity for a Week.

\begin{tabular}{lrr}
\hline Day & Quantity available & Quantity available \\
\hline Sunday & 100 & 112 \\
Monday & 50 & 48 \\
Tuesday & 60 & 60 \\
Wednesday & 40 & 38 \\
Thursday & 60 & 58 \\
Friday & 65 & 62 \\
Saturday & 80 & 83 \\
\hline
\end{tabular}


Table 3: Profitability Rating Considered with the Training Data.

\begin{tabular}{llr}
\hline State & Rating & Binary code \\
\hline High profit & $P 3+$ & 111 \\
Medium profit & $P 2+$ & 110 \\
Low profit & $P+$ & 101 \\
Profit & $P$ & 100 \\
Loss & $P-$ & 011 \\
Medium loss & $P 2-$ & 010 \\
Heavy loss & $P 3-$ & 001 \\
\hline
\end{tabular}

Table 4: Demand-Cost Profile for 7 Days with Profitability Rating $(P)$ for Training ANFIS.

\begin{tabular}{llll}
\hline Day & $\mathbf{P}_{1}$ & $\mathbf{P}_{2}$ & $\mathbf{P}_{3}$ \\
\hline Sunday & $P 3+$ & $P+$ & $P 3-$ \\
Monday & $P 3+$ & $P+$ & $P 3-$ \\
Tuesday & $P 2+$ & $P 2+$ & $P+$ \\
Wednesday & $P 2+$ & $P+$ & $P 2+$ \\
Thursday & $P 2+$ & $P+$ & $P 2+$ \\
Friday & $P 2+$ & $P+$ & $P-$ \\
Saturday & $P 3+$ & $P-$ & $P-$ \\
\hline
\end{tabular}

Table 5: Time and Average RMSE for 200 Epochs for ANFIS-Based DSS for Inventory Control Using 6 Weeks of Data with Variation in MF Types and Numbers.

\begin{tabular}{lrrr}
\hline Types of MF & No. of MFs & Time for 200 epochs (s) & RMSE after 200 epochs $\left(\times 10^{-3}\right)$ \\
\hline Bell MF & 3 & 1.93 & 2.72 \\
& 6 & 2.1 & 2.15 \\
& 9 & 2.2 & 2.2 \\
Gaussian MF & 3 & 1.8 & 2.6 \\
& 6 & 1.9 & 2.2 \\
& 9 & 2.0 & 1.8 \\
\hline
\end{tabular}

If the actual demand is more than the quantity available, the system triggers a decision state for supply and production; otherwise, the inventory is maintained and no order is placed for re-stocking. The training considerations also involve a certain profitability rating, which is combined with the day-to-day inventory records. Table 3 outlines the formulation of data relating the profitability rating with the demand-available cycle.

If the actual demand is more than the quantity available, the system triggers a decision state for supply and production. In state case, the mechanism for production and supply of items gets initiated. If the demand is less than the supply, the inventory is maintained and no order is placed for re-stocking. The training considerations also involve a certain profitability rating that is combined with the day-to-day inventory records. Tables 2 and 3 outline the formulation of data relating the profitability rating with the demand-available cycle. A demand-cost profile with the profitability rating is formed to train the ANFIS. This is shown in Table 4.

The states shown in Table 4 are codified and training executed using the data of a supply chain recorded over a number of weeks. During execution, the training time recorded is confined to $1.8-2.2 \mathrm{~s}$ for the sample sets. A total of 10-20 trials are performed for each of the sample sets, and the average results are considered. Some of the testing results are shown in Table 5.

The error curve for a 50-epoch training with 10 numbers of Gaussian MFs is shown in Figure 4. 


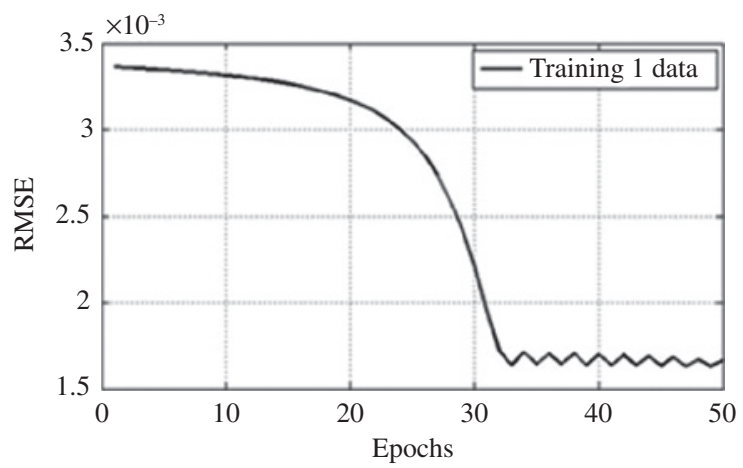

Figure 4: RMSE Plot for 50 Epochs with 10 Gaussian MFs.

The RMSE convergence is fast, which ensures an efficient decision making. For a 4-week data set, the readings have been found to be consistent. The above results are also compared with that of an ANN formulated in feed-forward form with two configurations of three hidden layers (15, 25, and 30 and 5, 12, and 16 numbers of log-sigmoidal, tan-sigmoidal, and log-sigmoidal activation functions). The first ANN with hidden layers $(15,25$, and 30$)$ is named $\mathrm{ANN}_{1}$, while the other with 5,12 , and 16 numbers of hidden layers is called $\mathrm{ANN}_{2}$. The results obtained from ANFIS-DSS are compared with those obtained from $\mathrm{ANN}_{2}$ and $\mathrm{ANN}_{2}$. The performance is measured in terms of epochs of training and accuracy achieved while making the decision. The summary of the results is depicted in Table 6.

While $\mathrm{ANN}_{1}$ and $\mathrm{ANN}_{2}$ take 15 and 18 epochs, respectively, to generate 87\% accuracy, the ANFIS takes 9 epochs to attain an $89 \%$ success rate while performing demand forecast of certain essential consumer items over a period of 4 weeks. While doing so, it takes 6 weeks of data. Similarly, during the second set of trials, with the same size of data, running 30 and 31 epochs, $\mathrm{ANN}_{1}$ and $\mathrm{ANN}_{2}$ generate success rates of $89 \%$ and $88 \%$, respectively, which the ANFIS exceeds with only 19 epochs and $92 \%$ accuracy generated. In another set of trials, the ANFIS takes 36 epochs to generate a $95 \%$ success rate by taking at least $67 \%$ less number of epochs compared to $\mathrm{ANN}_{1}$ and $\mathrm{ANN}_{1}$. This establishes the swift and correct decision-making abilities of the ANFIS, which is crucial in reducing production and supply costs. The proposed system has also been used to predict the demands of a certain consumer for several weeks. The AFNIS-based system consistently provided better accuracy. Figure 5 shows a set of results depicting the performance of the system in comparison to two ANNbased models using data obtained from a consumer goods supply chain.

Table 6: Comparison of Performance of ANFIS with ANN.

\begin{tabular}{lrr}
\hline Types & No. of epochs & \% Success rate \\
\hline ANN $_{1}$ & 15 & 87 \\
& 30 & 89 \\
& 45 & 92 \\
& 60 & 93 \\
& 73 & 93 \\
ANN $_{2}$ & 18 & 87 \\
& 31 & 88 \\
& 43 & 91 \\
& 43 & 92 \\
& 43 & 93 \\
ANFIS & 9 & 89 \\
& 19 & 91 \\
& 31 & 92 \\
& 36 & 95 \\
& 48 & 95 \\
\hline
\end{tabular}




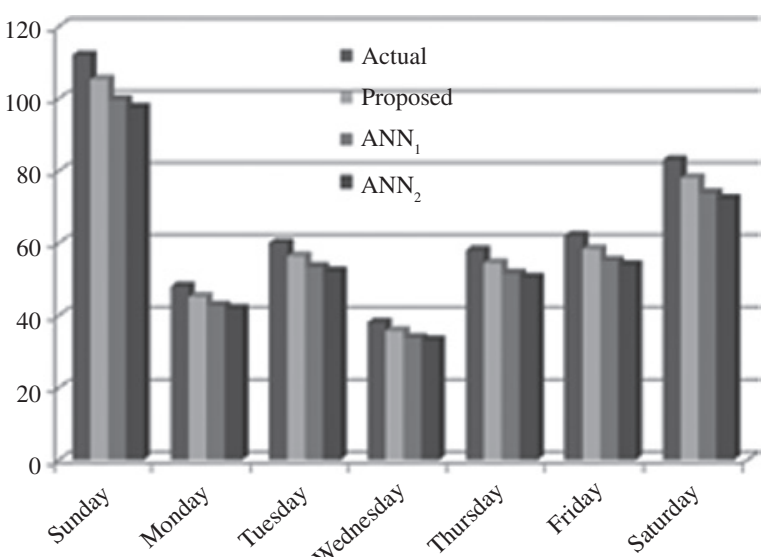

Figure 5: Prediction Accuracy of the ANFIS-Based System Compared to Two ANN-Based Approaches.

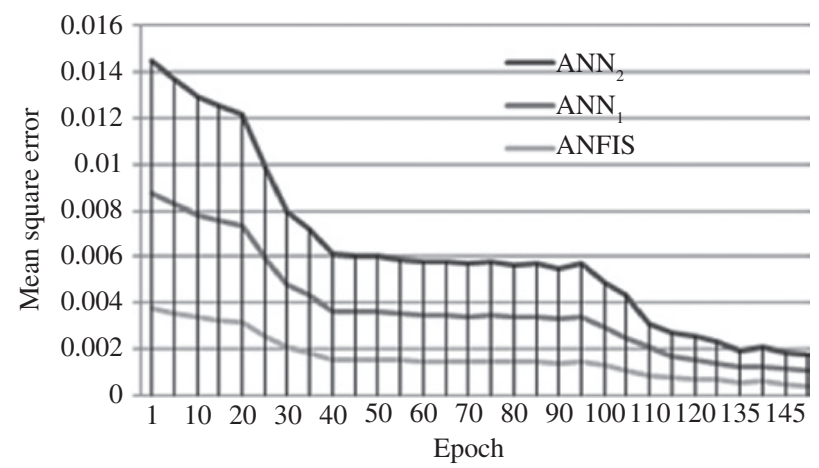

Figure 6: Mean Square Error (MSE) Variation with Epochs of the ANFIS-Based System Compared to Two ANN-Based Approaches.

Table 7: Comparison of Production and Supply Cost Savings in Comparison to EOQ Model by the Proposed ANFIS Method and ANNs.

\begin{tabular}{llrr}
\hline SI. No. & Method & Production cost saving & Supply cost saving \\
\hline 1 & $\mathrm{ANN}_{1}$ & $61 \%$ & $68 \%$ \\
2 & $\mathrm{ANN}_{2}$ & $63 \%$ & $70 \%$ \\
3 & $\mathrm{ANFIS}$ & $76 \%$ & $72 \%$ \\
\hline
\end{tabular}

The three techniques are trained with 2 months of data. The mean square errors of the ANFIS-based approach compared to $\mathrm{ANN}_{1}$ and $\mathrm{ANN}_{2}$ models are shown in Figure 6.

The effectiveness of the proposed system has also been tested in terms of savings achieved in production and supply costs compared to the standard EOQ model using 6 weeks data of an online fast-food supply concern. Table 7 summarizes the results in terms of cost savings generated by the ANFIS system compared to the traditional EOQ model [1].

The results establish the effectiveness of the proposed system.

\section{Conclusion}

Here, we have discussed the design of an ANFIS-based DSS for use as part of an inventory management system. The primary focus was to configure the ANFIS for generating a decision for 7 days of a week by 
including demand and supply on the one hand, and several other factors like procurement, ordering, and holding costs, to name a few, on the other hand. For the design of ANFIS, Gaussian MFs were used. The number of MFs used is also an important aspect. For ascertaining the training, the RMSE is considered to be an important factor. The performance was measured in terms of epochs of training and the accuracy achieved while making the decision. The results were compared to those obtained from an ANN as well, which also has learning-based decision-making capability. The experimental results established the advantage of use of ANFIS for the DSS, for use as part of an inventory management system.

\section{Bibliography}

[1] P. Aengchuan and B. Phruksaphanrat, Comparison of fuzzy inference system (FIS), FIS with artificial neural networks (FIS+ ANN) and FIS with adaptive neuro-fuzzy inference system (FIS + ANFIS) for inventory control, J. Intell. Manuf. (2015), 1-9, doi: 10.1007/s10845-015-1146-1.

[2] S. Agarwalla and K. K. Sarma, Machine learning based sample extraction for automatic speech recognition using dialectal Assamese speech, Neural Netw. 78 (2016), 97-111.

[3] S. A. Ahmed, S. Dey and K. K. Sarma, Image texture classification using artificial neural network (ANN), in: 2nd IEEE National Conference on Emerging Trends and Applications in Computer Science, Shillong, India, 2011.

[4] M. Baruah, A. Misra and K. K. Sarma, Split FTDNN-DFE equalizer for complex non-linear wireless channels with NARMA approximation, J. Intell. Syst. 26 (2016), 87-107.

[5] H. Bodoloi and K. K. Sarma, Protein structure prediction using multiple artificial neural network classifier, in: Soft Computing Techniques in Vision Science, Studies in Computational Intelligence, vol. 395, pp. 137-146, Springer-Verlag, 2012.

[6] H. C. Chang, An application of fuzzy sets theory to the EOQ model with imperfect quality items, Comput. Oper. Res. 31 (2004), 2079-2092.

[7] A. Choudhury, A. K. Talukdar, M. K. Bhuyan and K. K. Sarma, Movement epenthesis detection for continuous sign language recognition, J. Intell. Syst. 26 (2017), 471-481.

[8] T. Efendigil, S. Önüt and C. Kahraman, A decision support system for demand forecasting with artificial neural networks and neuro-fuzzy models: a comparative analysis, Exp. Syst. Appl. 36 (2009), 66-97.

[9] L. K. Gaafar and M. H. Choueiki, A neural network model for solving the lot-sizing problem, Omega 28 (2014), 175-184.

[10] J. Kacprzyk and P. Stanieski, Long-term inventory policy-making through fuzzy decision-making models, Fuzzy Sets Syst. 8 (2015), 117-132.

[11] Y. H. Lin, J. R. Shie and C. H. Tsai, Using an artificial neural network prediction model to optimize work-in-process inventory level for wafer fabrication, Exp. Syst. Appl. 36 (2009), 3421-3427.

[12] M. C. Lo, Decision support system for the integrated inventory model with general distribution demand, Inform. Technol. J. 6 (2007), 1069-1074.

[13] M. K. Maiti and M. Maiti, Fuzzy inventory model with two warehouses under possibility constraints, Fuzzy Sets Syst. 157 (2009), 52-73.

[14] K. S. Park, Fuzzy-set theoretic interpretation of economic order quantity, IEEE Trans. Syst. Man Cybernet. 17 (2012), 1082.

[15] S. Paul and A. Azeem, An artificial neural network model for optimization of finished goods inventory, Int. J. Indust. Eng. Comput. 2 (2011), 431-438.

[16] S. K. Paul and A. Azeem, Selection of the optimal number of shifts in fuzzy environment: manufacturing company's facility application, J. Indust. Eng. Manage. 3 (2012), 54-67.

[17] P. Pitchipoo, P. Venkumar and S. Rajakarunakaran, Modeling and development of a decision support system for supplier selection in the process industry, J. Indust. Eng. Int. 9 (2013), 1-15.

[18] K. K. Sarma and A. Mitra, Recurrent fuzzy-neural MIMO channel modeling, J. Intell. Syst. 21 (2012), 121-142.

[19] K. K. Sarma and A. Mitra, MIMO channel modeling using MLP with FIR and IIR synapses, IET Commun. 7 (2013), $1540-1549$.

[20] M. Sarma and K. K. Sarma, Vowel phoneme segmentation for speaker identification in Assamese using an ANN based framework, J. Intell. Syst. 22 (2013), 111-130.

[21] K. K. Sarma and N. Mastorakis, MIMO system with GA DFE-ANFIS framework, NAUN Int. J. Fuzzy Syst. Adv. Appl. 1 (2014), $55-60$.

[22] F. Sarwar, M. Rashid and D. Ghosh, An adaptive neuro-fuzzy inference system based algorithm for long term demand forecasting of natural gas consumption, in: Fourth International Conference on Industrial Engineering and Operations Management (IEOM, 2014), January 7-9, 2014.

[23] H. M. Wee, J. Yu and M. C. Chen, Optimal inventory model for items with imperfect quality and shortage back ordering, Omega 35 (2007), 7-11.

[24] L. A. Zadeh, Fuzzy sets, Inform. Control 8 (1965), 338-353. 